Document downloaded from:

http://hdl.handle.net/10251/55358

This paper must be cited as:

Stroeve, P.; Rahman, M.; Naidu, LD.; Chu, G.; Mahmoudi, M.; Ramirez Hoyos, P.; Mafé, S. (2014). Protein diffusion through charged nanopores with different radii at low ionic strength. Physical Chemistry Chemical Physics. 16(39):21570-21576. doi:10.1039/c4cp03198a.

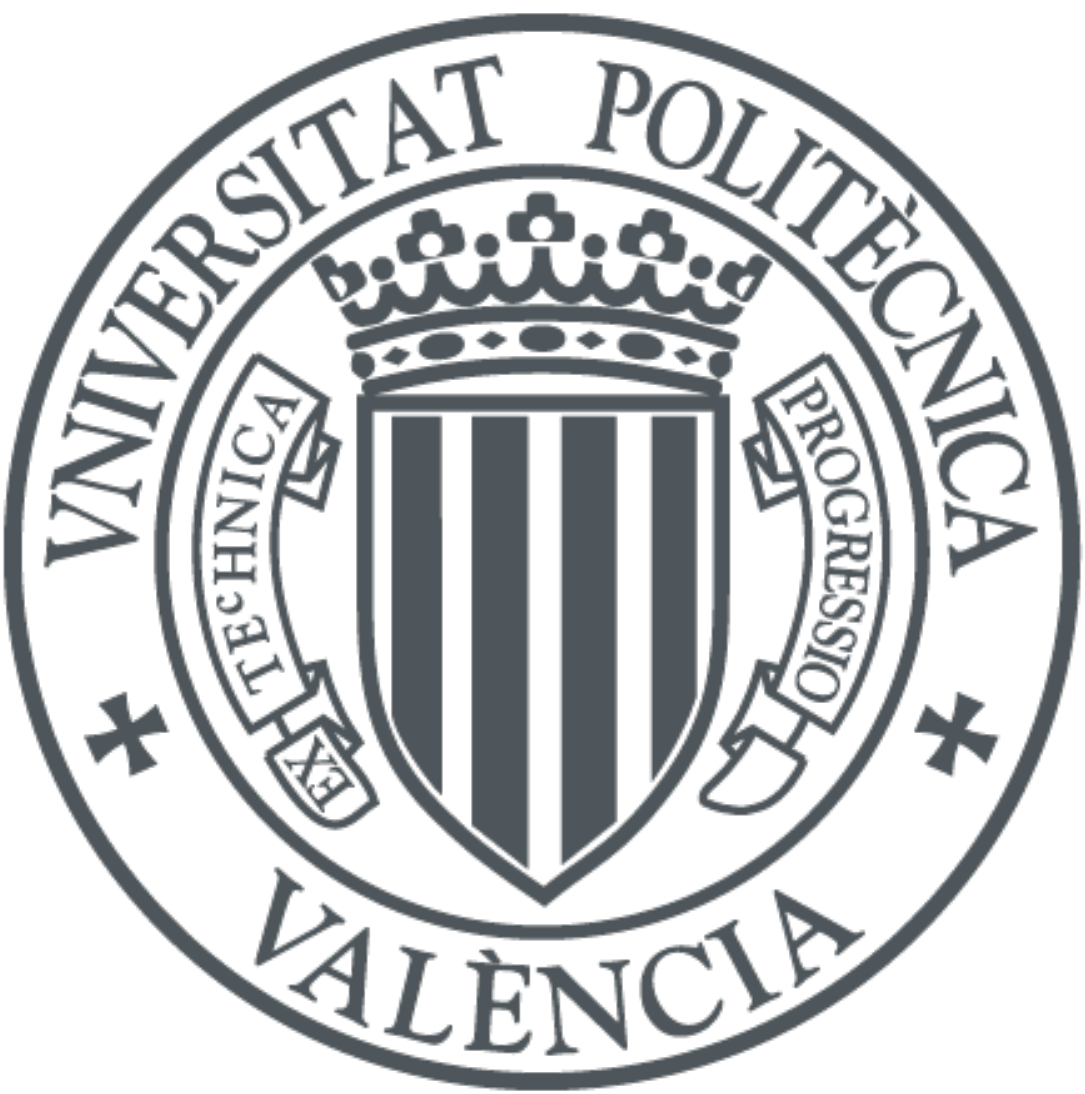

The final publication is available at

http://dx.doi.org/10.1039/c4cp03198a

Copyright Royal Society of Chemistry

Additional Information 


\title{
Protein diffusion through charged nanopores with different radii at low ionic strength
}

\author{
Pieter Stroeve, ${ }^{\mathrm{a}^{*}}$ Masoud Rahman, ${ }^{\mathrm{a}}$ Lekkala Dev Naidu, ${ }^{\text {a,b }}$ Gilbert Chu, ${ }^{\mathrm{a}}$ Morteza \\ Mahmoudi, ${ }^{\mathrm{c}}$ Patricio Ramirez, ${ }^{\mathrm{d}}$ and Salvador Mafe ${ }^{\mathrm{e}}$
}

The diffusion of two similar molecular weight proteins, bovine serum albumin (BSA) and bovine haemoglobin $(\mathrm{BHb})$, through nanoporous charged membranes with a wide range of pore radii is studied at low ionic strength. The effects of the solution $\mathrm{pH}$ and the membrane pore diameter on the pore permeability allow quantifying the electrostatic interaction between the charged pore and the protein. Because of the large screening Debye length, both surface and bulk diffusion occur simultaneously. By increasing the pore diameter, the permeability tends to the bulk self-diffusion coefficient for each protein. By decreasing the pore diameter, the charges on the pore surface electrostatically hinder the transport even at the isoelectric point of the protein. Surprisingly, even at pore sizes 100 times larger than the protein, the electrostatic hindrance still plays a major role in the transport. The experimental data are qualitatively explained using a two-region model for the membrane pore and approximated equations for the $\mathrm{pH}$ dependence of the protein and pore charges. The experimental and theoretical results should be useful for designing protein separation processes based on nanoporous charged membranes.

* Author to whom correspondence should be addressed: Dr. Pieter Stroeve, email: pstroeve@ucdavis.edu

\footnotetext{
${ }^{a}$ Department of Chemical Engineering and Materials Science, University of California Davis, Davis, CA 95616, USA

${ }^{b}$ National Institute of Technology Tiruchirapalli, Trichy 620015, India

${ }^{c}$ Nanotechnology Research Center, Faculty of Pharmaceutics, Tehran University of

Medical Sciences, Tehran, Iran

${ }^{\mathrm{d} D e p a r t m e n t}$ de Física Aplicada. Univ. Politècnica de València. E-46022 Valencia, Spain

${ }^{\mathrm{e}}$ Department de Física de la Terra i Termodinàmica, Universitat de València, E-46100 Burjassot, Spain
} 


\section{Introduction}

The transport of biomolecules through charged nanopores is a problem of fundamental and applied significance. Protein diffusion through nanoporous membranes is affected by a multitude of parameters such as osmotic pressure, ${ }^{1}$ electric field, ${ }^{2}$ membrane thickness, ${ }^{3}$ protein size (molecular weight), pore diameter, protein electrostatic charge and concentration, ${ }^{4}$ charge distribution and shape of the protein, pore shape and functional groups on the pore surface, ionic strength, ${ }^{5} \mathrm{pH}$, and temperature. In principle, the combination of these parameters may provide tools to control the transport of the protein. However, it is difficult to implement practical procedures when multiple effects are acting simultaneously and fundamental studies addressing only a limited number of effects under controlled conditions are of interest for designing protein separation processes.

Selecting the membrane pore size allows the separation of proteins with significantly different molecular sizes. In the case of proteins with nearly identical molecular weight, the separation requires the use of other parameters. ${ }^{6}$ Bovine serum albumin (BSA) has a molecular weight of $66,000 \mathrm{Da}$, an isoelectric point $\mathrm{p} I=4.7$, and a prolate ellipsoidal shape. The conformation of BSA does not change over a wide range of ionic strengths and $\mathrm{pH}^{7}$ Bovine hemoglobin $(\mathrm{BHb})$ has a molecular weight of $65,000 \mathrm{Da}$, an isoelectric point $\mathrm{p} I=7.0$, and a nearly spherical shape. We have shown previously for the case of narrow pores that, by adjusting the solution $\mathrm{pH}$ to the $\mathrm{p} I$ of one of the proteins, the transport of the other is hindered by the electrostatic interaction between the protein and the surface pore charges. ${ }^{5}$ Protein flux reaches a maximum at $\mathrm{p} I$ while the electrostatic interaction decreases this flux at higher or lower $\mathrm{pH}$ values. ${ }^{5}$

In addition to the $\mathrm{pH}$, the ionic strength of the solution can also modify the electrostatic interaction between the protein and the pore as well as the protein conformation. Note that the ionic strength dictates the Debye screening length in the solution. ${ }^{5,11}$ Additionally, an ionic strength difference on both sides of the membrane can increase protein transport. ${ }^{5}$ In those cases were the $\mathrm{pH}$ and ionic strength do not provide enough protein selectivity, membrane modification by functional groups and self-assembled monolayers can be used to enhance this selectivity. ${ }^{5}$ Polymeric membranes such as polycarbonate track-etched membranes (PCTE) have the advantages of being flexible, cost effective, commercially available for a wide range of pore diameters, and compatible with various acidic or basic environments. Inorganic membranes based on aluminum anodic oxide and silica films incorporate micro-arrays of pores that have lower thicknesses than many commercial polymer membranes. ${ }^{3}$ The different characteristics of these membranes have recently been reviewed. ${ }^{8}$ 
To better understand the electrostatic interactions between protein and membrane, we have studied here the effect of $\mathrm{pH}$ and pore size on the diffusive transport of $\mathrm{BHb}$ and BSA through non-modified PCTE membranes with different pore radii at low ionic strength. Because these interactions can be screened by the ions in solution, a relatively low ionic strength of 1 $\mathrm{mM}$ is used. Also, in order to reduce membrane fouling ${ }^{9}$ and diminish the interactions between the proteins, ${ }^{10}$ a low protein concentration (approximately $7 \mu \mathrm{M}$ ) is employed. We provide some basic clues to the problem of protein diffusion in charged nanoporous membranes. ${ }^{2,11-13}$ In narrow pores, the electrostatic interactions between the protein and the pore surface charges give low fluxes except for $\mathrm{pH}$ values close to $\mathrm{p} I$ where this interaction is decreased. ${ }^{1,2,5,11,12}$ This electrostatic hindrance is still operative for relatively large pore radii (compared with the protein radius) because at low ionic strength the Debye length can still cover a significant fraction of the pore.

\section{Materials and methods}

Polycarbonate membranes were purchased from Sterlitech. The hydraulic pore size of the membranes was measured using a method previously described. ${ }^{14}$ The experimental data varied around $\pm 20 \%$ from the specified pore size (nominal size) of the PCTE membranes. The nominal and the measured hydraulic pore diameters are summarised in Table 1. After measuring the hydraulic pore size, the same membrane was employed in all protein diffusion measurements for that particular pore size.

Proteins BSA and $\mathrm{BHb}$ were purchased from Sigma Aldrich. All the solutions were prepared at a fixed ionic strength of $0.001 \mathrm{M}$ using deionised water $(18.2 \mathrm{Mohm} / \mathrm{cm})$. The protein solutions were prepared at a fixed protein concentration of $0.5 \mathrm{mg} / \mathrm{ml}$. In order to adjust the $\mathrm{pH}$ of the solutions, $\mathrm{HCl}$ and $\mathrm{NaOH}$ were employed. The $\mathrm{NaCl}$ concentration required to obtain a fixed ionic strength was determined from the sum of concentrations $c_{\mathrm{HCl}}+c_{\mathrm{NaOH}}+c_{\mathrm{NaCl}}+\frac{1}{2} z^{2} c_{0}=0.001 \mathrm{M}$ for each particular case, where $c_{0}$ is the protein concentration in the reservoir volume and $z$ is the effective protein charge.

In the diffusion experiments, two solutions with the same $\mathrm{pH}$ and ionic strength were prepared. The first solution containing the protein was in the reservoir while the second solution without the protein was in the sink. The amount of protein needed to fix the ionic strength was obtained from the effective charge of the protein at each $\mathrm{pH}$. The effective charge of BSA was estimated from the dynamic NMR measurements of Böhme and Scheler. ${ }^{15}$ The effective charge of $\mathrm{BHb}$ as a function of the $\mathrm{pH}$ was estimated from the data by Berretta et al. ${ }^{16}$ It is important 
that both sides of the membrane have the same ionic strength because an ionic strength difference would influence the protein transport considerably. ${ }^{5}$

The diffusion apparatus was similar to that used previously. It consisted of a two compartment cell separated by the PCTE membrane. The reservoir compartment was filled with $30 \mathrm{ml}$ of protein solution and the sink compartment was filled with $30 \mathrm{ml}$ of solution of the same $\mathrm{pH}$ and ionic strength. The concentration of the protein in the sink compartment was measured every $10 \mathrm{~min}$ by UV-Visible spectroscopy. From the slope of the change of the protein concentration $c$ in the sink volume $V$ versus time, $(\Delta c / \Delta t)$, the total flux, the pore flux and the pore permeability $P$ were calculated. To this end, we used the mass balance equation $V(\Delta c / \Delta t)=P c_{0} A_{\mathrm{p}} / L_{\mathrm{p}}$, where $A_{\mathrm{p}}$ is the total pore area and $L_{\mathrm{p}}$ is the pore length. The measured hydraulic pore density and membrane thickness employed in the calculations of the pore flux and permeability are summarised in Table 1. All diffusion experiments were conducted at room temperature $\left(23^{\circ} \mathrm{C}\right)$.

After each diffusion experiment, the cell was washed and filled with a $0.1 \mathrm{M} \mathrm{NaOH}$ solution for one day. This procedure cleaned the membrane and preserved it. Before running the next experiment, both cell compartments were filled with the sink solution for one hour to equilibrate the membrane with the solution $\mathrm{pH}$. The solution compartments were magnetically stirred at $400 \mathrm{rpm}$. To prevent heat transfer from the magnetic stirrer to the diffusion cell, the cell was separated from the stirrer with a layer of foam insulation.

\section{Results and discussion}

\subsection{Experimental results}

Table 1 summarises relevant experimental data for the PCTE membranes used in the protein diffusion experiments. Figs. 1-3 show the pore permeability $P v s . \mathrm{pH}$ for proteins BSA and $\mathrm{BHb}$ in membranes with different pore diameters, as well as $P$ normalised to the permeability of the free solution $\left(P_{\mathrm{F}}\right)$ and to the permeability at the isoelectric point $\left(P_{0}\right)$. Fig. 1 shows that, for the case of the large pores, the maximum values of $P$ for proteins $\mathrm{BHb}\left(0.71 \times 10^{-}\right.$ $\left.{ }^{6} \mathrm{~cm}^{2} / \mathrm{s}\right)$ and BSA $\left(0.62 \times 10^{-6} \mathrm{~cm}^{2} / \mathrm{s}\right)$ occur at their respective isoelectric points $\mathrm{p} I$. For the sake of comparison, Fig. 2 shows $P / P_{\mathrm{F}} v s . \mathrm{pH}$ while Fig. 3 shows $P / P_{0} v s . \mathrm{pH}$.

The protein transport data in terms of the pore permeability $P$ are not dependent on the membrane thickness and the concentration difference across the membrane, which permits the comparison between different membranes. The measured permeabilities for BSA and BHb in Fig. 1 show a significant increase of $P$ with the pore diameter, as expected. Also, the maximum 
pore permeability at $\mathrm{p} I$ shows that the electrostatic interaction with the pore charges is relatively small when the protein net charge is zero. ${ }^{5}$ By increasing the pore diameter, the permeability at the isoelectric point becomes closer to the self-diffusion coefficient $D$ of $\mathrm{BSA}^{17}$ and $\mathrm{BHb}^{10,18}$ in a free solution, as shown in Fig. 2. This suggests to write $P$ as proportional to $D$ through a dimensionless hindrance factor $K, P=K D$. Therefore, $K$ tends to unity when the pore diameter increases because the protein transport for large pores is similar to bulk diffusion (hydrodynamic and electrostatic hindrances become very small in the case of large pores, as shown in Figs. 2 and 3).

By decreasing the pore diameter, the role of the electrostatic hindrance on diffusion becomes more important, as shown in Fig. 3. Note however that the electrostatic interaction between the protein and the pore is still significant even for large pore diameters (about 100 times larger than the protein size) close to the isoelectric point. We will consider this question in the next section.

\subsection{Model calculations}

We aim at describing theoretically the effect of the protein and pore charges on diffusion through nanopores. We have studied previously a similar problem for the case of narrow pores (diameters of the order of $10 \mathrm{~nm}$ ) and moderately high electrolyte concentrations where surface effects are dominant. ${ }^{2}$ We consider here the case of large pores (diameters of the order of $100 \mathrm{~nm}$ ) and low electrolyte concentration where both surface and bulk diffusion are significant. To this end, we assume a two-region approach for the cylindrical pore (Fig. 4) together with simple titration models for the protein and pore charges. ${ }^{2,19-25}$

The pore surface is covered by a $\mathrm{pH}$-dependent fixed charge density $\sigma$ described by the equilibrium dissociation ${ }^{22}$

$$
\sigma=\frac{\sigma_{\mathrm{m}}}{1+10^{\mathrm{p} K_{\mathrm{a}}-\mathrm{pH}}}
$$

where $\sigma_{\mathrm{m}}$ is the maximum surface charge density and the $\mathrm{p} K_{\mathrm{a}}$ corresponds to carboxylic acid groups. Around the respective isoelectric points, the average protein charge numbers are estimated as

$$
z=z_{0}+\frac{N_{+}}{1+10^{\mathrm{pH}-\mathrm{p} K_{\mathrm{a}+}}}
$$


$z=z_{0}-\frac{N_{-}}{1+10^{\mathrm{p} K_{\mathrm{a}-}-\mathrm{pH}}}+\frac{N_{+}}{1+10^{\mathrm{pH}-\mathrm{p} K_{\mathrm{a}+}}}$

Eq. (2a) corresponds approximately to the charge distribution of human $\mathrm{Hb}$ in the $\mathrm{pH}$ range $6<$ $\mathrm{pH}<8 .^{2,23}$ This model protein has $N_{+}=24$ basic residues with a common $\mathrm{p} K_{\mathrm{a}+}=7$ plus a constant number of negatively charged acid residues with a total charge $z_{0}=-10$. Ignoring the interaction between neighbouring residues and the effect of ionic strength on the protein charge, Eq. (2a) gives an isoelectric point around $7 .^{23}$ Eq. (2b) corresponds approximately to BSA, with $z_{0}=73, N_{-}=100$ and $N_{+}=16$, and the effective values ${ }^{21} \mathrm{p} K_{\mathrm{a}_{-}}=4.2$ and $\mathrm{p} K_{\mathrm{a}^{+}}=6.9$. Eq. (2b) gives an isoelectric point around 5. As a first approximation, the pH values in Eqs. (2a) and (2b) are assumed to be those of the external solutions. All the above literature data are compiled in Table 2.

Because of the high uncertainties concerning the geometry and charge distributions of the protein and nanopore, we estimate the protein flux density $J$ relative to the maximum achieved at the isoelectric point, $J_{0}$, rather than the absolute flux density. Hindrance factors due to the protein and nanopore radii $^{26}$ allow then to obtain $J_{0}$ in terms of the free diffusion value $J_{\mathrm{F}}$ (in general, $J<J_{0}<J_{\mathrm{F}}$ ). For the central pore region of Fig. 4, we assume that electrostatic effects are almost negligible and the protein flux density is approximately equal to $J_{0}$ (isoelectric point) at all $\mathrm{pH}$ values. In principle, this assumption should be reasonable because the surface charges are effectively screened by the ionic solution when the pore radius is much larger than the Debye length (see Table 2 and Fig. 4). On the contrary, electrostatic effects are dominant for diffusion over the surface pore region. Therefore, the protein flux density $J_{\mathrm{S}}$ due to surface diffusion should be much lower than $J_{0}$ for $\mathrm{pH}$ values different from $\mathrm{p} I$ (the protein and the pore are charged in this case).

The surface diffusion of Fig. 4 is an activated process relatively slow compared with the bulk diffusion in the pore center. ${ }^{19}$ As a first approximation, we assume that protein surface diffusion involves two processes that should occur sequentially. The protein has to enter first the surface region (in) from the central region and has to leave then the surface region (out) to the central region (Fig. 4). The charges on the pore surface act as effective barriers for these processes. $^{2,19}$ If the charges $\sigma$ (pore) and $z$ (protein) have the same sign, the electrostatic repulsion makes it difficult the first process (in) because of the protein exclusion from the pore surface. On the contrary, if $z$ and $\sigma$ have different signs, the protein may be adsorbed to the pore surface and the second process (out) is now inhibited. Therefore, one of these two processes should be rate limiting for surface diffusion. 
The effective rate constant describing the in and out processes occurring in series should be proportional to $k_{\text {in }} k_{\text {out }} /\left(k_{\text {in }}+k_{\text {out }}\right)$, with $k_{\text {in }} / k_{0}=\exp \left(-z e \psi_{\mathrm{s}} / k T\right)=k_{0} / k_{\text {out }}$, where $\psi_{\mathrm{s}}$ and $k_{0}$ are the surface electric potential due to the pore charges and the rate constant for $\psi_{\mathrm{S}}=0$, respectively. The constants $e, k$, and $T$ are the elementary charge, the Boltzmann constant, and the temperature, respectively. As a first approximation to the electrostatics problem, we consider point ions for the electrolyte solution and the linearised Poisson-Boltzmann (P-B) equation for a charged plane, so that $\psi_{\mathrm{S}} \approx \sigma L_{\mathrm{D}} / \varepsilon$ where $\varepsilon$ is the water electrical permittivity. The above assumptions are based on the fact that the pore radius is much larger than the Debye screening length and the protein diameter $\left(R>>L_{\mathrm{D}}>r\right.$ in Fig. 4 for most experimental cases). Note also that the measured zeta-potentials are usually lower than $k T / e=26 \mathrm{mV}$ in these pores due to the low charge density $\sigma,{ }^{22}$ which justifies further the use of the linearised P-B equation.

The protein flux density $J_{\mathrm{S}}$ should be proportional to the effective rate constant defined above and can then be written as

$\frac{J_{\mathrm{S}}}{J_{0}}=\frac{2}{\exp \left(z e \sigma L_{\mathrm{D}} / \varepsilon k T\right)+\exp \left(-z e \sigma L_{\mathrm{D}} / \varepsilon k T\right)}$

The values of $z$ and $\sigma$ in Eq. (3) should be evaluated at each pH making use of Eqs. (1) and (2). Eq. (3) gives the maximum value $J_{\mathrm{S}}=J_{0}$ at the isoelectric point where $z(\mathrm{pH}=\mathrm{p} I)=0$. On the contrary, as soon as the $\mathrm{pH}$ departs from $\mathrm{p} I$, one of the two exponential terms in the denominator of Eq. (3) takes high values and thus $J_{\mathrm{S}} \approx 0$. The surface pore region in Fig. 4 is then unavailable for transport because of either the protein exclusion $(\mathrm{pH}>\mathrm{p} I)$ from the surface region or the protein adsorption $(\mathrm{pH}<\mathrm{p} I)$ to this surface. Note that the effect of protein adsorption to charged surfaces could be significant ${ }^{27,28}$ Although the concentration in these experiments is low, electrostatic interactions will exist between the pore wall and protein molecules.

From the two pore regions of Fig. 4 , the total flux density $J$ relative to the maximum value $J_{0}$ is

$$
J / J_{0}=\left[1-\left(1-L_{\mathrm{D}} / R\right)^{2}\right] J_{\mathrm{s}} / J_{0}+\left(1-L_{\mathrm{D}} / R\right)^{2}
$$

Note that the flux density $J_{0}$ occurs over the whole pore section at the isoelectric point. This flux can then be written in terms of the free diffusion (bulk) flux density $J_{\mathrm{F}}$ by introducing the Renkin factor for hindered diffusion: ${ }^{26}$

$$
J_{0} / J_{\mathrm{F}}=F(r / R), \quad F(r / R)=(1-r / R)^{2}\left[1-2.1(r / R)+2.09(r / R)^{3}-0.95(r / R)^{5}\right]
$$


Eqs. (1)-(5) constitute an admittedly crude description of the formidable transport problem. More detailed microscopic models for the two-region pore and the electrostatic equations can be used at the price of increasing complexity. ${ }^{20,29,30}$ In particular, Zidney and coworkers $^{30-32}$ have also identified the (protein charge)-(pore charge) interaction in the exponentials of Eq. (3) as a crucial term in more rigorous models of protein ultrafiltration. Biesheuvel and co-workers ${ }^{20}$ have analysed thoroughly protein adsorption and partition equilibrium in charged nanopores. Renaud and co-workers ${ }^{29}$ have provided experimental and theoretical evidences for the transport transition between the surface-dominated and bulk diffusion regimes of Fig. 4 in the case of planar nanochannels with controlled geometry (see, e.g., Fig. 4 of Ref. 29). However, we will consider here only qualitative estimations and assume that the experimental permeability ratio $P / P_{\mathrm{F}}$ in Fig. 2 should behave similarly as the flux ratio $J / J_{\mathrm{F}}$ obtained from Eqs. (4) and (5).

Fig. 5 shows that the above model describes qualitatively the observed experimental trends of Fig. 2: the electrostatic interactions between the protein and the pore lead to lower flux densities for charged than for neutral proteins $\left(J / J_{\mathrm{F}}\right.$ peaks at $\left.\mathrm{pH}=\mathrm{p} I\right)$. This is a direct consequence of the exponentials in the denominator of Eq. (3) showing the interplay between the protein and nanopore charges. ${ }^{2}$ The theoretical maxima are more marked than the experimental ones because of the high protein charge obtained theoretically when $\mathrm{pH}$ departs from $\mathrm{p} I$. However, it is well known that not all of the protein charges are effective: the ionic screening gives an effective protein charge lower than the net charge obtained from the titration curves (see, e.g., Ref. 15 for the case of BSA). This effect is absent in Eqs. (2a) and (2b) but it can be incorporated in the model by arbitrarily decreasing the value of $z .{ }^{15}$ The dashed curves of Fig. 5 are obtained with an effective value $z_{\text {eff }}<z$ and, as expected, this procedure gives less marked maxima, in agreement with Fig. 2. Note also that the model captures the asymmetric behaviour of BSA around $p I$, which is due to the asymmetric charge distribution given by the protein model (Eq. (2b)) and the progressive neutralisation of the pore as $\mathrm{pH}$ tends to $\mathrm{p} K_{\mathrm{a}-}=3.9$ (Eq. (1)).

Although the above model provides some qualitative clues to this complex transport problem, it is based on a reduced number of assumptions that should be justified. The division of the pore into two geometrical regions of significantly different transport properties (Fig. 4) may be plausible when the screening Debye length is significant but does not cover all the pore section. ${ }^{29}$ However, this procedure introduces some uncertainties concerning the relative sizes of the pore regions in Fig. 4. Assuming a continuous radial variation of the pore properties ${ }^{29,30,33}$ would be more realistic for quantitative estimations but it is difficult in our case because of the limited microscopic information on the protein-pore surface interaction. 
Note also that Eq. (3) is a crude estimation for the protein surface transport that oversimplifies the pore electrostatics and considers only the effect of the protein net charge. Localised patches of non-zero charge on a globally neutral protein surface may still interact electrostatically with the pore charges even at $\mathrm{p} I$. Therefore, not only the protein net charge but also the heterogeneous charge distribution and particular shape characteristic of each protein should be important. ${ }^{34-37}$ Although the incorporation of the protein geometrical shape and microscopic surface charge distribution is far beyond the qualitative model used here, these details could be significant at the isoelectric point, producing a hindrance effect even at $\mathrm{p} I^{5,11}$ Indeed, the experimental data show that the protein hindered diffusion at $\mathrm{p} I$ cannot be described with the Renkin function only (Fig. 6), especially for the pores with low radii. This fact suggests that the surface pore region of Fig. 4 should not be completely available for protein transport even at $\mathrm{p} I$. To describe qualitatively this effect, Fig. 6 shows that reducing the effective pore radius from $R$ to $R-L_{\mathrm{D}}$ leads to a new hindrance factor $F\left(r /\left(R-L_{\mathrm{D}}\right)\right)<F(r / R)$ which better follows the experimental permeabilities.

The Renkin's correction (Eq. (5)) gives the correct asymptotic value for the higher values of $(R / r)$ but it fails to describe the experimental data when $(R / r)$ decreases down to 10 in Fig. 6. Alternatively, we may adopt a modified version of the Brenner and Gaydos ${ }^{38}$ classical approach and write:

$$
J_{0} / J_{\mathrm{F}}=\left\{\left[1-r /\left(R-L_{\mathrm{D}}\right)\right]^{2} /[1-r / R]^{2}\right\}[1-(9 / 8)(r / R) \ln (R / r)-1.539(r / R)]
$$

In Eq. (6), only the partition coefficient has been modified to reflect the exclusion of the protein from a region within a distance $L_{\mathrm{D}}$ of the pore wall (the hydrodynamic drag contributed by the pore wall has not been changed ${ }^{38}$ ). Eq. (6) gives the dotted-dashed curve of Fig. 6 showing a slightly better agreement with the experimental data than Eq. (5).

\section{Conclusions}

Diffusion experiments have been conducted with nanoporous charged membranes for proteins $\mathrm{BSA}$ and $\mathrm{BHb}$. The effects of the solution $\mathrm{pH}$ and the membrane pore diameter have been described in terms of the pore permeability, which constitutes a method to quantify the electrostatic interaction between the charged pore and the protein. We have considered the case of large pores (diameters of the order of $100 \mathrm{~nm}$ ) and low electrolyte concentrations where both surface and bulk diffusion are expected to occur simultaneously. By increasing the pore diameter, the pore permeability tends to the free self-diffusion coefficient for both proteins. Interestingly, the transport is electrostatically hindered by the charges on the pore surface even 
at the protein isoelectric point when the pore radius is comparable with the screening Debye length. The experimental data are qualitatively explained using a two-region model for the pore together with simple titration equations for the protein and pore charges. The basic clues provided should be useful for protein separation processes based on nanoporous charged membranes.

\section{Acknowledgements}

We thank the UCOP Lab Fee Program for financial support. P. R. and S. M. acknowledge the financial support from the Ministry of Economy and Competitiveness of Spain and FEDER (project MAT2012-32084) and the Generalitat Valenciana (project PROMETEO/GV/0069).

\section{References}

1. $\quad$ N. S. Pujar and A. L. Zydney, J. Chromatogr. A 1998, 796, 229-238.

2. K.-Y. Chun, S. Mafé, P. Ramírez and P. Stroeve, Chem. Phys. Lett. 2006, 418, 561-564.

3. N. Ileri, R. Faller, A. Palazoglu, S. E. Letant, J. W. Tringe and P. Stroeve, PCCP 2013, 15, 965-71.

4. D. B. Burns and A. L. Zydney, AIChE J. 2001, 47, 1101-1114.

5. $\quad$ K.-Y. Chun and P. Stroeve, Langmuir 2002, 18, 4653-4658.

6. H. U. Osmanbeyoglu, T. B.Hur and H. K. Kim, J. Membrane Sci. 2009, 343, 1-6.

7. C. Tanford and J. G.Buzzell, J. Phys. Chem. 1956, 60, 225-231.

8. P. Stroeve and N. Ileri, Trends in Biotech. 2011, 29, 259-66.

9. C.-C. Ho and A. L. Zydney, Ind. Eng. Chem. Res. 2001, 40, 1412-1421.

10. V. Riveros-Moreno and J. B. Wittenberg, J. Biol. Chem. 1972, 247, 895-901.

11. J.-R. Ku and P. Stroeve, Langmuir 2004, 20, 2030-2032.

12. S. Yu, S. B. Lee, M. Kang and C.R. Martin, Nanolett 2001, 1, 495-497.

13. S. Yu, S. B. Lee, M. Kang and C. R. Martin, Anal. Chem. 2003, 75, 1239-1244

14. Z. Hou, N. L. Abbott and P. Stroeve, Langmuir 2000, 16, 2401-2404.

15. U.Böhme and U. Scheler, Chem. Phys. Lett. 2007, 435, 342-345.

16. S. Beretta, G. Chirico, D. Arosio and G. Baldini, Macromolecules 1997, 30, 7849-7855.

17. A. K. Gaigalas, J. B. Hubbard, M. McCurley and S. Woo, J. Phys. Chem. 1992, 96, 2355-2359.

18. K. LaGattuta, V. Sharma, D. Nicoli and B. Kothari, Biophys. J. 1981, 33, 63-79. 
19 S. Mafe, J. A. Manzanares and P. Ramirez, Phys. Chem. Chem. Phys. 2003, 5, 376-383.

20 P. Biesheuvel, P. Stroeve and P. Barneveld, J. Phys. Chem. B 2004, 108, 17660-17665.

21 P. Biesheuvel and A. Wittemann, J. Phys. Chem. B 2005, 109, 4209-4214.

22 W. H. Keesom R. L. Zelenka and C. J. Radke, J. Colloid Interface 1988, 125, 575-585.

23 G. B. Benedek and F. M. H. Villars, Physics with illustrative examples from Medicine and Biology (Statistical Physics), Springer-Verlag, Heidelberg, 2000.

24 D. Arosio, H. E. Kwansa, H. Gering, G. Piszczek and E. Bucci, Biopolymers 2002, 63, $1-11$.

25 I. Axelsson, J. Chromatogr. A 1978, 152, 21-32.

26 R. E. Beck and J. S. Schultz, Science 1970, 170, 1302-1305.

27 D. B. Burns and A. L. Zidney, Biotechnol. Bioeng. 1999, 64, 27-37.

28 R. B. Schoch, A. Bertsch and P. Renaud, Nano Lett. 2006, 6, 543-547.

29 N. F.Y. Durand, C. Dellagiacoma, R. Goetschmann, A. Bertsch, I. Märki, T. Lasser and P. Renaud, Anal. Chem. 2009, 81, 5407-5412.

30 M.M. Rohani and A.L. Zydney, Adv. Colloid Interface Sci. 2010, 160, 40-48.

31 M. K. Menon and A. L. Zydney, Biotechnol. Bioeng. 1999, 63, 298-307.

32 M. M. Rohani and A. L. Zydney, J. Membrane Sci. 2009, 337, 324-331.

33 S. Mafe, J. A. Manzanares and J. Pellicer, J. Membrane Sci. 1990, 51, 161-168.

34 J. C. Bosma and J. A. Wesseling, AIChE J. 1998, 44, 2399-2409.

35 Q. Shi, Y. Zhou and Y. Sun, Biotechnol. Prog. 2005, 21, 516-523.

36 B. Jönsson and J. Stahlberg, Colloids Surf. B. 1999, 14, 67-75.

37 Q. Shi, Y. Zhou and Y. Sun, Biotechnol. Prog. 2005, 21, 516-523.

38 H. Brenner and L. J. Gaydos, J. Colloid Interf. Sci. 1977, 58, 312-356. 


\begin{tabular}{|c|c|c|c|c|c|c|c|}
\hline $\begin{array}{l}\text { Nominal pore } \\
\text { diameter }(\mathrm{nm})\end{array}$ & 30 & 50 & 80 & 100 & $400^{\$}$ & $800^{*}$ & $800^{*}$ \\
\hline $\begin{array}{l}\text { Measured } \\
\text { hydraulic pore } \\
\text { diameter }(\mathrm{nm})\end{array}$ & 57 & 70 & 96 & 116 & 332 & 665 & 837 \\
\hline $\begin{array}{ll}\text { Nominal } & \text { pore } \\
\text { density }^{* *} & \left(10^{8}\right. \\
\left.\text { pores } / \mathrm{cm}^{2}\right) & \end{array}$ & 6 & 6 & 4 & 4 & 1 & 0.3 & 0.3 \\
\hline $\begin{array}{l}\text { Membrane } \\
\text { thickness }^{* *} \\
(\mu \mathrm{m})\end{array}$ & 6 & 6 & 6 & 6 & 10 & 9 & 9 \\
\hline \multicolumn{8}{|c|}{$\begin{array}{l}\left.{ }^{\$}\right) \text { Another } 400 \mathrm{~nm} \text { nominal membrane had a } 488 \mathrm{~nm} \text { measured hydraulic pore diameter, but was used } \\
\text { for only one BSA experiment because inadvertently the membrane was damaged after the experiment } \\
\text { *) Two membranes with a nominal diameter of } 800 \mathrm{~nm} \text { were employed to determine the protein } \\
\text { permeability for large pore sizes. } \\
{ }^{* *} \text { ) These data are based on the manufacturer specifications for each membrane. }\end{array}$} \\
\hline
\end{tabular}

Table 1 Characteristics of the PCTE membranes used in the protein diffusion experiments. 
Protein

Charge $z(\mathrm{pH})$ of $\mathrm{BHb}$ in Eq. (2a)

23

Charge $z(\mathrm{pH})$ of BSA in Eq. (2b)

20,21

Protein radius $r=3.5 \mathrm{~nm}$

24,25

Pore

Pore charge $\sigma(\mathrm{pH})$ in Eq. (1)

22

Maximum surface charge $\sigma_{\mathrm{m}}=-0.002 \mathrm{C} / \mathrm{m}^{2}$

22

$\mathrm{p} K_{\mathrm{a}}$ (carboxylic groups) $=3.9$

22

Pore diameters $2 R=56,140$, and $560 \mathrm{~nm}$

\section{Ionic solution}

Equivalent ionic concentration of the aqueous solution: $1 \mathrm{mM}$

Water electrical permittivity $\varepsilon=79 \varepsilon_{0}, \varepsilon_{0}=8.8510^{-12} \mathrm{C} /(\mathrm{V} \mathrm{m})$

Debye length $L_{\mathrm{D}}=10 \mathrm{~nm}$

Table 2 The protein, pore and solution characteristics together with the source references. 

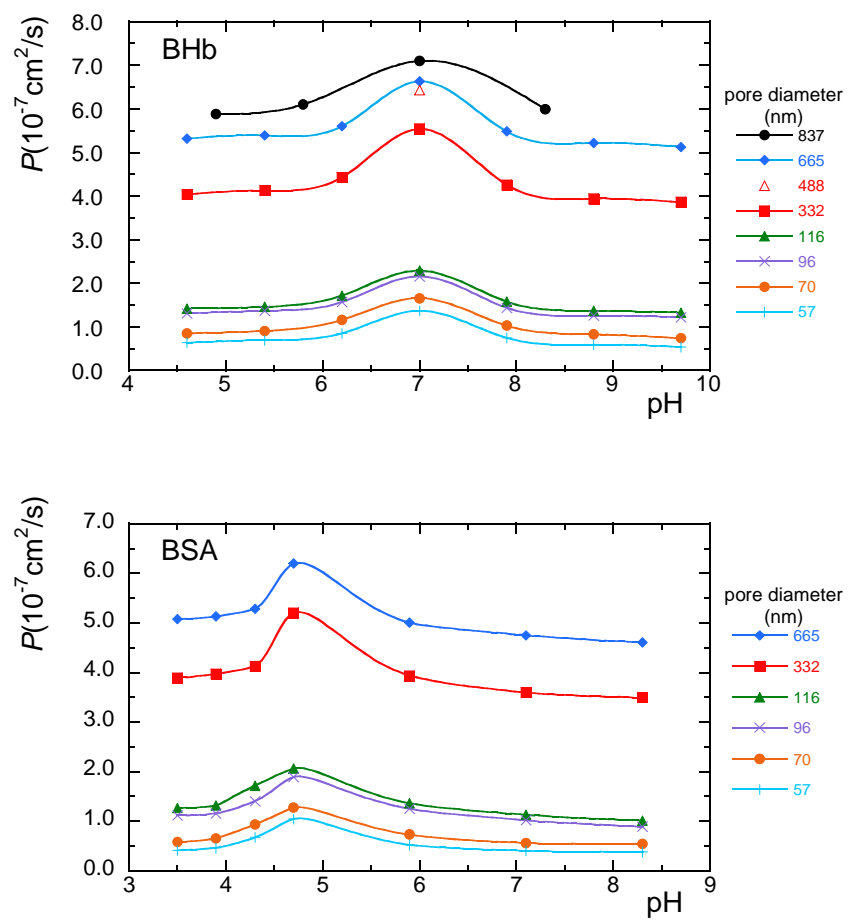

Fig. 1 Pore permeability $P$ vs. $\mathrm{pH}$ for proteins $\mathrm{BSA}$ and $\mathrm{BHb}$ in membranes with different pore diameters. For the case of the large pores, the maximum values of $P$ occurring at the isoelectric points of BHb and BSA are close to the respective bulk diffusion coefficients. 

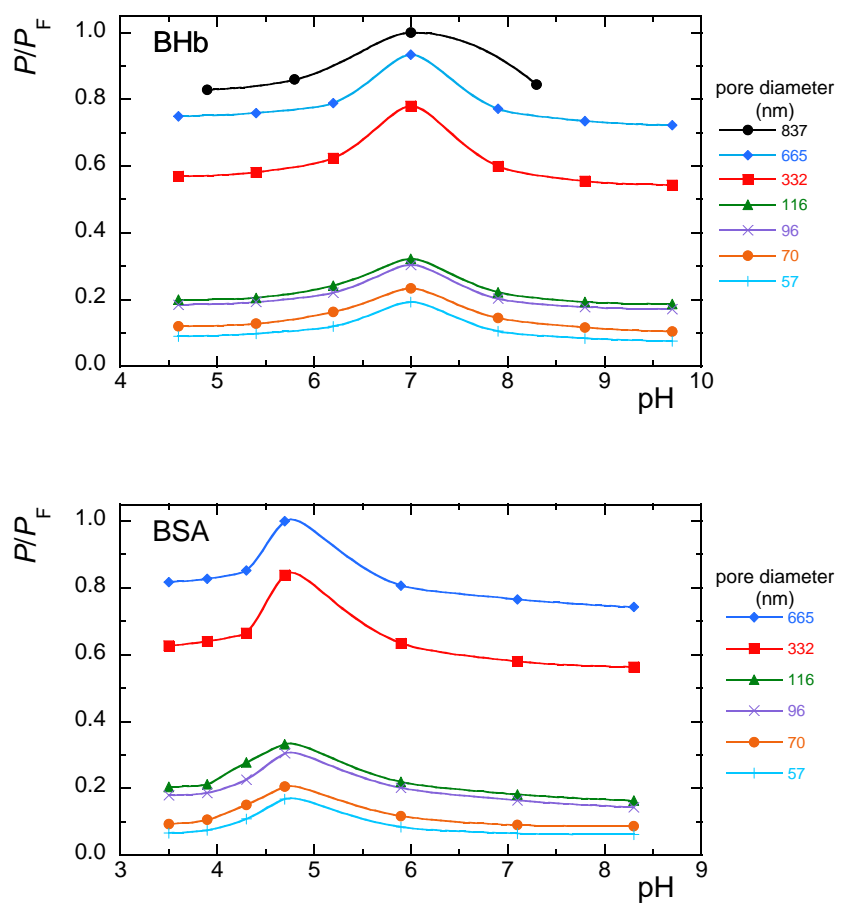

Fig. 2 Pore permeability $P$ (normalised to the pore permeability of the free solution, $P_{\mathrm{F}}$ ) $v s . \mathrm{pH}$ for different pore diameters. Note that $P_{\mathrm{F}}$ corresponds to the bulk diffusion coefficient of the protein since the distribution coefficient is equal to one. 

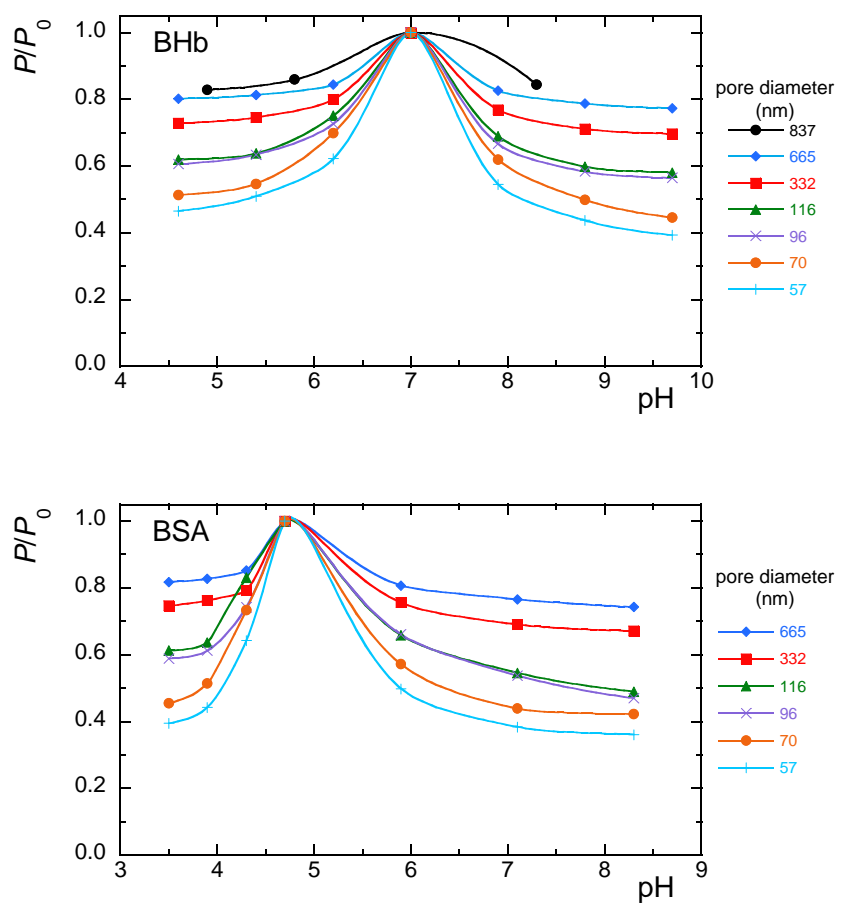

Fig. 3 Pore permeability $P$ (normalised to the pore permeability at the isoelectric point, $P_{0}$, for each pore diameter) $v s . \mathrm{pH}$ for membranes with different pore diameters. 


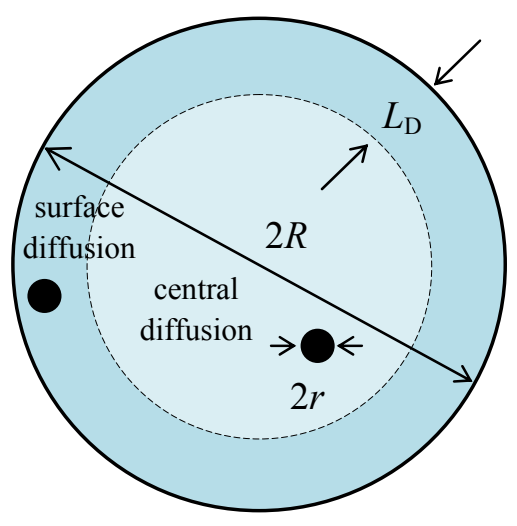

Fig. 4 Two-region model for a pore of diameter $2 R$ (not to scale). Electrostatically hindered surface diffusion occurs over the surface pore region of thickness $L_{\mathrm{D}}$, with $L_{\mathrm{D}}=10 \mathrm{~nm}$ for an equivalent electrolyte concentration of $0.001 \mathrm{M}$. Bulk diffusion proceeds over the central pore region of radius $R-L_{\mathrm{D}}$. The model protein (black sphere) has a diameter $2 r=7 \mathrm{~nm}$ (note that the typical Stokes radii of proteins ${ }^{24,25} \mathrm{BHb}$ and BSA are between 3.2 and $3.5 \mathrm{~nm}$ ). In the transport experiments, the pore radius $R>L_{\mathrm{D}}>2 r$. 

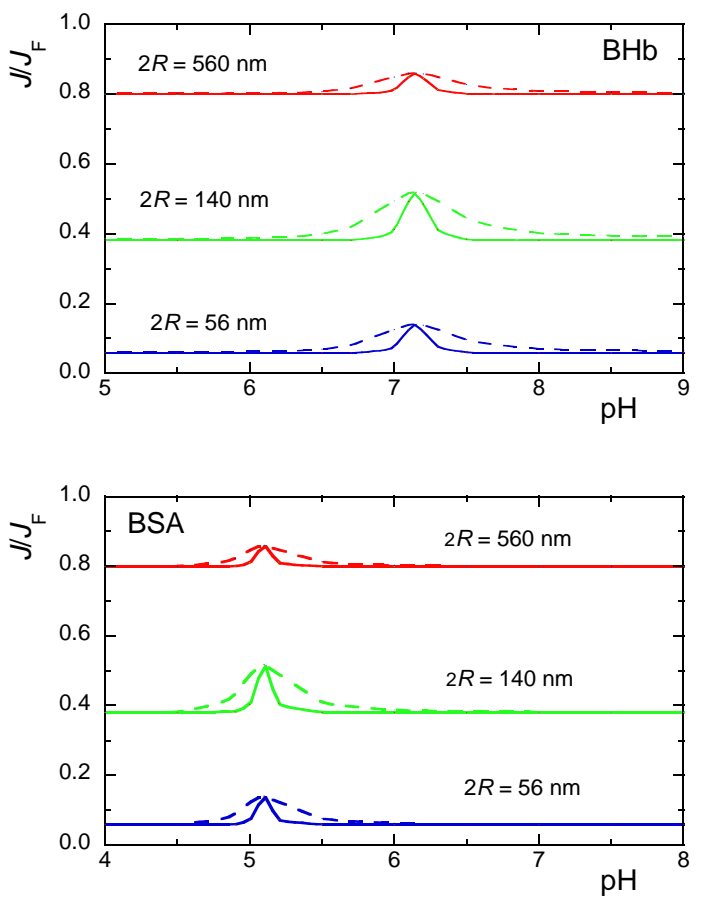

Fig. 5 The ratio $J / J_{\mathrm{F}} v s$. $\mathrm{pH}$ calculated from Eqs. (4) and (5) for model proteins $\mathrm{BHb}$ (up) and BSA (bottom) at three pore radii. The dashed curves show the effect of ionic screening giving an effective protein charge $z_{\text {eff }}<z$. We consider $z_{\text {eff }}=0.3 z$, where $z$ is obtained from Eqs. (2a) and $(2 b)$. 


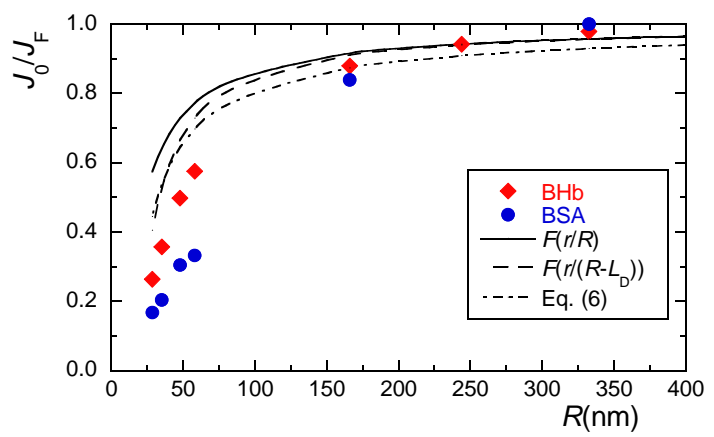

Fig. 6 The ratio of the flux density $J_{0}$ at the isoelectric point to the free diffusion flux density $J_{\mathrm{F}}$ is approximated by the Renkin factor for hindered diffusion (Eq. (5) with $r=3.5 \mathrm{~nm}$ ). This factor is calculated assuming that the surface pore region of thickness $L_{\mathrm{D}}=10 \mathrm{~nm}$ is available (continuous curve) and unavailable (dashed curve) for protein transport at $\mathrm{p} I$. In the second case, the effective pore radius should be $R-L_{\mathrm{D}}$ instead of $R$, and then surface diffusion occurs in the pore region between $R-2 L_{\mathrm{D}}$ and $R-L_{\mathrm{D}}$ in Fig. 4 . The dotted-dashed curve is obtained with Eq. (6) for the same values of $r$ and $L_{\mathrm{D}}$ and the points correspond to the experimental data for proteins $\mathrm{BHb}$ and BSA. 